\title{
Generalized Synchronization of Nonlinear Chaotic Systems through Natural Bioinspired Controlling Strategy
}

\author{
Shih-Yu Li, ${ }^{1,2}$ Shi-An Chen, ${ }^{1}$ Chin-Teng Lin, ${ }^{1,2}$ Li-Wei Ko, ${ }^{1,3}$ \\ Cheng-Hsiung Yang, ${ }^{4}$ and Heng-Hui Chen ${ }^{5}$ \\ ${ }^{1}$ Brain Research Center, National Chiao Tung University, Hsinchu, Taiwan \\ ${ }^{2}$ Department of Electrical and Computer Engineering, National Chiao Tung University, Hsinchu, Taiwan \\ ${ }^{3}$ Department of Biological Science and Technology, National Chiao Tung University, 1001 Ta Hsueh Road, Hsinchu 300, Taiwan \\ ${ }^{4}$ Graduate Institute of Automation and Control, National Taiwan University of Science and Technology, Taipei, Taiwan \\ ${ }^{5}$ Department of Mechanical Engineering, Hsiuping University of Science and Technology, Taichung, Taiwan
}

Correspondence should be addressed to Shi-An Chen; cuteless.ece93g@gmail.com

Received 10 November 2014; Revised 20 March 2015; Accepted 21 March 2015

Academic Editor: Jinde Cao

Copyright (C) 2015 Shih-Yu Li et al. This is an open access article distributed under the Creative Commons Attribution License, which permits unrestricted use, distribution, and reproduction in any medium, provided the original work is properly cited.

\begin{abstract}
A novel bioinspired control strategy design is proposed for generalized synchronization of nonlinear chaotic systems, combining the bioinspired stability theory, fuzzy modeling, and a novel, simple-form Lyapunov control function design of derived high efficient, heuristic and bioinspired controllers. Three main contributions are concluded: (1) apply the bioinspired stability theory to further analyze the stability of fuzzy error systems; the high performance of controllers has been shown in previous study by Li and Ge 2009, (2) a new Lyapunov control function based on bioinspired stability theory is designed to achieve synchronization without using traditional LMI method, which is a simple linear homogeneous function of states and the process of designing controller to synchronize two fuzzy chaotic systems becomes much simpler, and (3) three different situations of synchronization are proposed; classical master and slave Lorenz systems, slave Chen's system, and Rossler's system as functional system are illustrated to further show the effectiveness and feasibility of our novel strategy. The simulation results show that our novel control strategy can be applied to different and complicated control situations with high effectiveness.
\end{abstract}

\section{Introduction}

Since Pecora and Carroll first obtained the synchronization of two identical chaotic systems under different initial conditions [1], synchronization of chaotic systems has received significant attention. Nowadays, more and more techniques of chaos synchronization were proposed, such as active control [2-4], backstepping control method [5-8], linear error feedback control [9-11], adaptive control [12-16], and sliding mode control [17-20].

In our real world, various kinds of nonlinear chaotic biosystems describing natural phenomenon are found to have some states always positive. It means these states are always in the first quadrant, such as the three species prey-predator systems [15, 16, 21], double Mackey-Glass systems [17, 22, 23], energy communication system in biological research [19, 24], and virus-immune system [20]. For the three species preypredator systems, which consist of two competing preys and one predator can be described by the following set of nonlinear differential equations:

$$
\begin{aligned}
& \frac{d x}{d T}=r_{1}\left(1-k_{1}^{-1} x-k_{1}^{-1} c_{2} y\right)-\Phi_{1}(x, y) z, \\
& \frac{d y}{d T}=r_{2}\left(1-k_{2}^{-1} c_{1} x-k_{2}^{-1} y\right)-\Phi_{2}(x, y) z, \\
& \frac{d z}{d T}=e_{1} \Phi_{1}(x, y) z+e_{2} \Phi_{2}(x, y) z-\alpha z,
\end{aligned}
$$

where $\alpha, r_{i}, k_{i}, e_{i}$, and $c_{i}, i=1,2$ are the model parameters assuming only positive values, and the functions $\Phi_{i}(x, y)$, 
$i=1,2$ represent the densities of the two prey species and $z$ represents the density of the predator species. The predator $z$ consumes the preys $x, y$ according to the response functions [25]. The prescribed model characterized by nonlinear response since amount of food consumed by predator per unit time depends upon the available food sources from the two preys $x$ and $y$. And for the virus-immune system, a mathematical model is considered as follows:

$$
\begin{aligned}
\frac{d T}{d t} & =s-\mu_{1} T+r T\left[1-\frac{T+I}{\Gamma}\right]-k V T, \\
\frac{d I}{d t} & =k V T-\mu_{2} I, \\
\frac{d V}{d t} & =\mu_{3} N I-k V T-\mu_{4} V,
\end{aligned}
$$

where $T, I$, and $V$ represent the population concentrations of uninfected, infected target cells and virus, respectively. We denote by the $s$ constant supply of target cells from its precursor, where $s$ is the rate at which new T cells are created. These cells have finite life time and $\mu_{1}$ represent the average death rates of these cells, $\mu_{2}$ and $\mu_{3}$ represent the death rate of the infected cells before viral production commences, and $\mu_{4}$ is the viral clearance rate constant. These target cells are assumed to grow logistically with specific growth rate $r$ and carrying capacity $\Gamma$. Above all, their states are all positive.

As a result, a natural bioinspired stability theory is proposed for generalized synchronization in this paper. It means that there exists a given functional relationship between the states of the master and that of the slave. Via using the natural bioinspired stability theory, the new Lyapunov function is a simple linear homogeneous function of states and introduces less simulation error.

In this paper, a new Lyapunov control function based on the bioinspired stability theory is proposed to synchronize fuzzy chaotic systems. This new Lyapunov control function is designed as a simple linear homogeneous function of states and the process of designing controller to synchronize two fuzzy chaotic systems becomes much simpler. Furthermore, we design three different synchronization situations to show the effectiveness of our strategy-(1) CASE I, synchronization of identical systems: synchronization of master fuzzy Lorenz system and slave fuzzy Lorenz system, (2) CASE II, synchronization of two different systems: synchronization of master fuzzy Lorenz system and slave fuzzy Chen system, and (3) CASE III, synchronization of complicated different systems: synchronization of master fuzzy Lorenz system and complicated fuzzy chaotic system (slave fuzzy Chen system and functional fuzzy Rossler's system).

The layout of the rest of the paper is as follows. In Section 2, the natural bioinspired stability theory is introduced. In Section 3, modeling of chaotic systems is proposed. In Section 4, three simulation examples are given. In Section 5, conclusions are given.

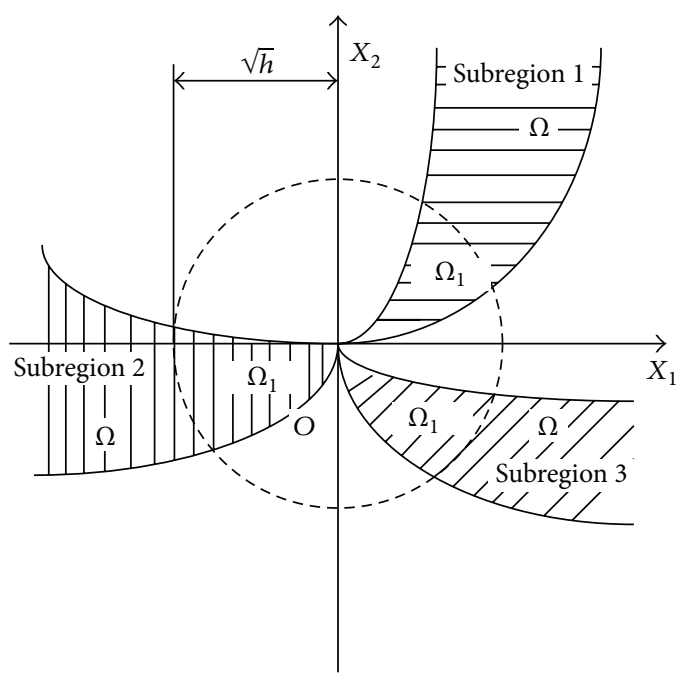

FIGURE 1: Bioinspired partial regions $\Omega$ and $\Omega_{1}$.

\section{Natural Bioinspired Controlling Strategy}

2.1. Definition of the Stability on Partial Region. Consider the differential equations of disturbed motion of a nonautonomous system in the normal form

$$
\frac{d x_{s}}{d t}=X_{s}\left(t, x_{1}, \ldots, x_{n}\right), \quad(s=1, \ldots, n),
$$

where the function $X_{s}$ is defined on the intersection of the partial region $\Omega$ (shown in Figure 1) and

$$
\sum_{s} x_{s}^{2} \leq H
$$

and $t>t_{0}$, where $t_{0}$ and $H$ are certain positive constants. $X_{s}$, which vanishes when the variables $x_{s}$ are all zero, is a real valued function of $t, x_{1}, \ldots, x_{n}$. It is assumed that $X_{s}$ is smooth enough to ensure the existence and uniqueness of the solution of the initial value problem. When $X_{s}$ does not contain $t$ explicitly, the system is autonomous.

Obviously, $x_{s}=0(s=1, \ldots, n)$ is a solution of (3). We are interested in the asymptotical stability of this zero solution on partial region $\Omega$ (including the boundary) of the neighborhood of the origin which in general may consist of several subregions (Figure 1).

Definition 1. For any given number $\varepsilon>0$, if there exists a $\delta>0$, such that on the closed given partial region $\Omega$ when

$$
\sum_{s} x_{s 0}^{2} \leq \delta, \quad(s=1, \ldots, n)
$$

for all $t \geq t_{0}$, the inequality

$$
\sum_{s} x_{s}^{2}<\varepsilon, \quad(s=1, \ldots, n)
$$

is satisfied for the solutions of (3) on $\Omega$, then the disturbed motion $x_{s}=0(s=1, \ldots, n)$ is stable on the partial region $\Omega$. 
Definition 2. If the undisturbed motion is stable on the partial region $\Omega$, and there exists a $\delta^{\prime}>0$, so that on the given partial region $\Omega$ when

$$
\sum_{s} x_{s 0}^{2} \leq \delta^{\prime}, \quad(s=1, \ldots, n)
$$

the equality

$$
\lim _{t \rightarrow \infty}\left(\sum_{s} x_{s}^{2}\right)=0
$$

is satisfied for the solutions of (3) on $\Omega$, then the undisturbed motion $x_{s}=0(s=1, \ldots, n)$ is asymptotically stable on the partial region $\Omega$.

The intersection of $\Omega$ and region defined by (7) is called the region of attraction.

Definition of Functions $V\left(t, x_{1}, \ldots, x_{n}\right)$. Let us consider the functions $V\left(t, x_{1}, \ldots, x_{n}\right)$ given on the intersection $\Omega_{1}$ of the partial region $\Omega$ and the region

$$
\sum_{s} x_{s}^{2} \leq h, \quad(s=1, \ldots, n)
$$

for $t \geq t_{0}>0$, where $t_{0}$ and $h$ are positive constants. We suppose that the functions are single-valued and have continuous partial derivatives and become zero when $x_{1}=$ $\cdots=x_{n}=0$.

Definition 3. If there exists $t_{0}>0$ and a sufficiently small $h>0$, so that on partial region $\Omega_{1}$ and $t \geq t_{0}, V \geq 0$ (or $\leq 0$ ), then $V$ is a positive (or negative) semidefinite, in general semidefinite, function on the $\Omega_{1}$ and $t \geq t_{0}$.

Definition 4. If there exists a positive (negative) definitive function $W\left(x_{1} \cdots x_{n}\right)$ on $\Omega_{1}$, so that on the partial region $\Omega_{1}$ and $t \geq t_{0}$

$$
\begin{array}{r}
V-W \geq 0 \\
\text { or }-V-W \geq 0,
\end{array}
$$

then $V\left(t, x_{1}, \ldots, x_{n}\right)$ is a positive definite function on the partial region $\Omega_{1}$ and $t \geq t_{0}$.

Definition 5. If $V\left(t, x_{1}, \ldots, x_{n}\right)$ is neither definite nor semidefinite on $\Omega_{1}$ and $t \geq t_{0}$, then $V\left(t, x_{1}, \ldots, x_{n}\right)$ is an indefinite function on partial region $\Omega_{1}$ and $t \geq t_{0}$. That is, for any small $h>0$ and any large $t_{0}>0, V\left(t, x_{1}, \ldots, x_{n}\right)$ can take either positive or negative value on the partial region $\Omega_{1}$ and $t \geq t_{0}$.

Definition 6 (bounded function $V$ ). If there exists $t_{0}>0, h>$ 0 , so that on the partial region $\Omega_{1}$, we have

$$
\left|V\left(t, x_{1}, \ldots, x_{n}\right)\right|<L,
$$

where $L$ is a positive constant, then $V$ is said to be bounded on $\Omega_{1}$.
Definition 7 (function with infinitesimal upper bound). If $V$ is bounded, and for any $\lambda>0$, there exists $\mu>0$, so that on $\Omega_{1}$ when $\sum_{s} x_{s}^{2} \leq \mu$, and $t \geq t_{0}$, we have

$$
\left|V\left(t, x_{1}, \ldots, x_{n}\right)\right| \leq \lambda
$$

then $V$ admits an infinitesimal upper bound on $\Omega_{1}$.

\subsection{Bioinspired GYC Theorem of Stability and of Asymptotical Stability on Partial Region}

Theorem 8. If there can be found a definite function $V\left(t, x_{1}, \ldots, x_{n}\right)$ on the partial region for (3) and the derivative with respect to time based on these equations is

$$
\frac{d V}{d t}=\frac{\partial V}{\partial t}+\sum_{s=1}^{n} \frac{\partial V}{\partial x_{s}} X_{s}
$$

then, it is a semidefinite function on the partial region whose sense is opposite to that of $V$, or if it becomes zero identically, then the undisturbed motion is stable on the partial region.

Proof. Let us assume for the sake of definiteness that $V$ is a positive definite function. Consequently, there exists a sufficiently large number $t_{0}$ and a sufficiently small number $h<H$, such that on the intersection $\Omega_{1}$ of partial region $\Omega$ and

$$
\sum_{s} x_{s}^{2} \leq h, \quad(s=1, \ldots, n)
$$

and $t \geq t_{0}$, the following inequality is satisfied

$$
V\left(t, x_{1}, \ldots, x_{n}\right) \geq W\left(x_{1}, \ldots, x_{n}\right) \text {, }
$$

where $W$ is a certain positive definite function which does not depend on $t$. Besides that, (13) may assume only negative or zero value in this region.

Let $\varepsilon$ be an arbitrarily small positive number. We will suppose that in any case $\varepsilon<h$. Let us consider the aggregation of all possible values of the quantities $x_{1}, \ldots, x_{n}$, which are on the intersection $\omega_{2}$ of $\Omega_{1}$ and

$$
\sum_{s} x_{s}^{2}=\varepsilon
$$

and let us designate by $l>0$ the precise lower limit of the function $W$ under this condition. By virtue of (10), we will have

$$
V\left(t, x_{1}, \ldots, x_{n}\right) \geq l \text { for }\left(x_{1}, \ldots, x_{n}\right) \text { on } \omega_{2} \text {. }
$$

We will now consider the quantities $x_{s}$ as functions of time which satisfy the differential equations of disturbed motion. We will assume that the initial values $x_{s 0}$ of these functions for $t=t_{0}$ lie on the intersection $\Omega_{2}$ of $\Omega_{1}$ and the region

$$
\sum_{s} x_{s}^{2} \leq \delta
$$


where $\delta$ is so small that

$$
V\left(t_{0}, x_{10}, \ldots, x_{n 0}\right)<l .
$$

By virtue of the fact that $V\left(t_{0}, 0, \ldots, 0\right)=0$, such a selection of the number $\delta$ is obviously possible. We will suppose that in any case the number $\delta$ is smaller than $\varepsilon$. Then the inequality

$$
\sum_{s} x_{s}^{2}<\varepsilon
$$

being satisfied at the initial instant will be satisfied, in the very least, for a sufficiently small $t-t_{0}$, since the functions $x_{s}(t)$ very continuously with time. We will show that these inequalities will be satisfied for all values $t>t_{0}$. Indeed, if these inequalities were not satisfied at some time, there would have to exist such an instant $t=T$ for which this inequality would become an equality. In other words, we would have

$$
\sum_{s} x_{s}^{2}(T)=\varepsilon,
$$

and consequently, on the basis of (19)

$$
V\left(T, x_{1}(T), \ldots, x_{n}(T)\right) \geq l .
$$

On the other hand, since $\varepsilon<h$, the inequality (9) is satisfied in the entire interval of time $\left[t_{0}, T\right]$, and consequently, in this entire time interval $d V / d t \leq 0$. This yields

$$
V\left(T, x_{1}(T), \ldots, x_{n}(T)\right) \leq V\left(t_{0}, x_{10}, \ldots, x_{n 0}\right),
$$

which contradicts (20) on the basis of (19). Thus, the inequality (23) must be satisfied for all values of $t>t_{0}$.

Finally, we must point out that from the view-point of mathematics, the stability on partial region in general does not be related logically to the stability on whole region. If an undisturbed solution is stable on a partial region, it may be either stable or unstable on the whole region and vice versa. In specific practical problems, we do not study the solution starting within $\Omega_{2}$ and running out of $\Omega$.

Theorem 9. If in satisfying the conditions of Theorem 8 the derivative $d V / d t$ is a definite function on the partial region with opposite sign to that of $V$ and the function $V$ itselfpermits an infinitesimal upper limit, then the undisturbed motion is asymptotically stable on the partial region.

Proof. Let us suppose that $V$ is a positive definite function on the partial region and that, consequently, $d V / d t$ is negative definite. Thus on the intersection $\Omega_{1}$ of $\Omega$ and the region defined by (9) and $t \geq t_{0}$ there will be satisfied not only the inequality (10), but also the following inequality:

$$
\frac{d V}{d t} \leq-W_{1}\left(x_{1}, \ldots, x_{n}\right),
$$

where $W_{1}$ is a positive definite function on the partial region independent of $t$.

Let us consider the quantities $x_{s}$ as functions of time which satisfy the differential equations of disturbed motion assuming that the initial values $x_{s 0}=x_{s}\left(t_{0}\right)$ of these quantities satisfy the inequalities (18). Since the undisturbed motion is stable in any case, the magnitude $\delta$ may be selected so small that for all values of $t \geq t_{0}$ the quantities $x_{s}$ remain within $\Omega_{1}$. Then, on the basis of (24) the derivative of function $V\left(t, x_{1}(t), \ldots, x_{n}(t)\right)$ will be negative at all times and, consequently, this function will approach a certain limit, as $t$ increases without limit, remaining larger than this limit at all times. We will show that this limit is equal to some positive quantity different from zero. Then for all values of $t \geq t_{0}$ the following inequality will be satisfied:

$$
V\left(t, x_{1}(t), \ldots, x_{n}(t)\right)>\alpha,
$$

where $\alpha>0$.

Since $V$ permits an infinitesimal upper limit, it follows from this inequality that

$$
\sum_{s} x_{s}^{2}(t) \geq \lambda, \quad(s=1, \ldots, n),
$$

where $\lambda$ is a certain sufficiently small positive number. Indeed, if such a number $\lambda$ did not exist, that is, if the quantity $\sum_{s} x_{s}(t)$ was smaller than any preassigned number no matter how small, then the magnitude $V\left(t, x_{1}(t), \ldots, x_{n}(t)\right)$, as follows from the definition of an infinitesimal upper limit, would also be arbitrarily small, which contradicts (25).

If for all values of $t \geq t_{0}$ the inequality (26) is satisfied, then (24) shows that the following inequality will be satisfied at all times:

$$
\frac{d V}{d t} \leq-l_{1}
$$

where $l_{1}$ is positive number different from zero which constitutes the precise lower limit of the function $W_{1}\left(t, x_{1}(t), \ldots, x_{n}(t)\right)$ under condition (26). Consequently, for all values of $t \geq t_{0}$ we will have

$$
\begin{aligned}
V & \left(t, x_{1}(t), \ldots, x_{n}(t)\right) \\
& =V\left(t_{0}, x_{10}, \ldots, x_{n 0}\right)+\int_{t_{0}}^{t} \frac{d V}{d t} d t \\
& \leq V\left(t_{0}, x_{10}, \ldots, x_{n 0}\right)-l_{1}\left(t-t_{0}\right),
\end{aligned}
$$

which is, obviously, in contradiction with (25). The contradiction thus obtained shows that the function $V\left(t, x_{1}(t), \ldots, x_{n}(t)\right)$ approached zero as $t$ increase without limit. Consequently, the same will be true for the function $W\left(x_{1}(t), \ldots, x_{n}(t)\right)$ as well, from which it follows directly that

$$
\lim _{t \rightarrow \infty} x_{s}(t)=0, \quad(s=1, \ldots, n),
$$

which proves the theorem.

Discussion and Statement. Theorems 8 and 9 in Bioinspired GYC Theorem of Stability of Asymptotical Stability on Partial Region provides a new way to investigate the stability problem-to solve the stability problem in the first quadrant, 
called Partial Region, which is inspired via the biological behavior in nature and is used to control the states of a system which exist in the first quadrant to achieve the goal system with a set of simple controllers through designing a simpler and more convenient Lyapunov function.

\section{Modeling of Chaotic Systems}

In this section, the well-famous Takagi-Sugeno fuzzy model is applied to model the classical Lorenz system, Chen system, and Rossler's system for further designing the natural bioinspired controller.

3.1. Fuzzy Modeling of Master Lorenz System. For master Lorenz system

$$
\begin{aligned}
& \dot{x}_{1}=a\left(x_{2}-x_{1}\right), \\
& \dot{x}_{2}=c x_{1}-x_{1} x_{3}-x_{2}, \\
& \dot{x}_{3}=x_{1} x_{2}-b x_{3},
\end{aligned}
$$

where $a, b, c$ are the parameters. When $a=10, b=8 / 3$, $c=28$, and initial states are $(0.5,1,5)$, the dynamic behavior is chaotic. Assume that $x_{1} \in\left[-d_{1}, d_{1}\right]$ and $d_{1}>0$; then Lorenz system can be exactly represented by T-S fuzzy model as follows:

Rule 1: IF $x$ is $M_{1}$, THEN $\dot{X}(t)=A_{1} X(t)$,

Rule 2: IF $x$ is $M_{2}$, THEN $\dot{X}(t)=A_{2} X(t)$,

where

$$
\begin{aligned}
X & =\left[x_{1}, x_{2}, x_{3}\right]^{T}, \\
A_{1} & =\left[\begin{array}{ccc}
-a & a & 0 \\
c & -1 & -d_{1} \\
0 & d_{1} & -b
\end{array}\right], \\
A_{2} & =\left[\begin{array}{ccc}
-a & a & 0 \\
c & -1 & d_{1} \\
0 & -d_{1} & -b
\end{array}\right], \\
M_{1}(x) & =\frac{1}{2}\left(1+\frac{x_{1}}{d_{1}}\right), \\
M_{2}(x) & =\frac{1}{2}\left(1-\frac{x_{1}}{d_{1}}\right),
\end{aligned}
$$

and $d_{1}=30 . M_{1}$ and $M_{2}$ are fuzzy set of Lorenz system. Here, we call (31) the first liner subsystem under the fuzzy rule and (32) the second liner subsystem under the fuzzy rule. The final output of the fuzzy Lorenz system is inferred as follows and the chaotic behavior is shown in Figure 2

$$
\dot{X}(t)=\sum_{i=1}^{2} H_{i} A_{i} X(t)
$$

TABLE 1

\begin{tabular}{lcc}
\hline System & Parameters & Initial states \\
\hline Fuzzy Lorenz & $a=10, b=8 / 3$, & $\left(x_{1}, x_{2}, x_{3}\right)=(0.5,1,5)$ \\
system (master) & $c=28, d_{1}=30$ & \\
\hline
\end{tabular}

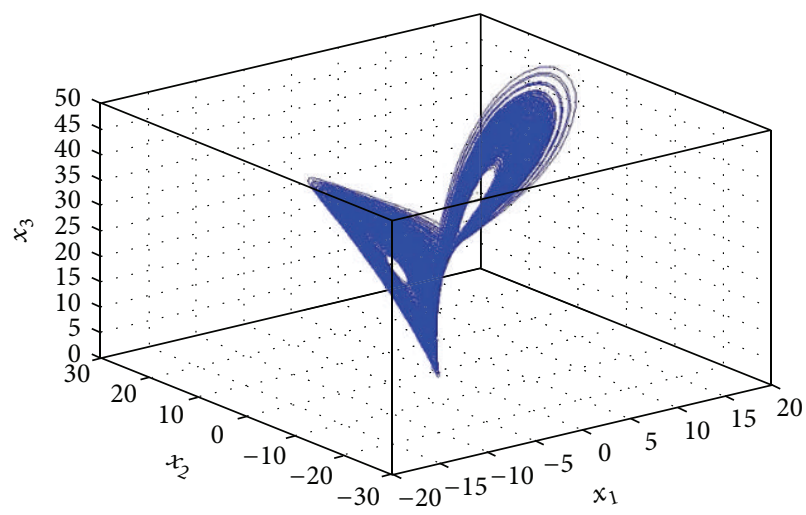

FIGURE 2: Chaotic behavior of master fuzzy Lorenz system.

where

$$
\begin{aligned}
& H_{1}=\frac{M_{1}}{M_{1}+M_{2}}, \\
& H_{2}=\frac{M_{2}}{M_{1}+M_{2}} .
\end{aligned}
$$

We further summarize all the parameters in Section 3.1 into Table 1 .

3.2. Fuzzy Modeling of Slave Lorenz System. For slave Lorenz system

$$
\begin{aligned}
& \dot{y}_{1}=a\left(y_{2}-y_{1}\right)+u_{1}, \\
& \dot{y}_{2}=c y_{1}-y_{1} y_{3}-y_{2}+u_{2}, \\
& \dot{y}_{3}=y_{1} y_{2}-b y_{3}+u_{3},
\end{aligned}
$$

where $a, b, c$ are the parameters. When $a=10, b=8 / 3$, $c=28$, and initial states are $(0.6,3,10)$, the dynamic behavior is chaotic. Assume that $y_{1} \in\left[-d_{2}, d_{2}\right]$ and $d_{2}>0$; then Lorenz system can be exactly represented by T-S fuzzy model as follows:

Rule 1: IF $y$ is $\widetilde{M}_{1}$, THEN $\dot{Y}(t)=\widetilde{A}_{1} Y(t)+U_{1}$,

Rule 2: IF $y$ is $\widetilde{M}_{2}, \operatorname{THEN} \dot{Y}(t)=\widetilde{A}_{2} Y(t)+U_{2}$,

where

$$
\begin{aligned}
Y & =\left[y_{1}, y_{2}, y_{3}\right]^{T}, \\
\widetilde{A}_{1} & =\left[\begin{array}{ccc}
-a & a & 0 \\
c & -1 & -d_{2} \\
0 & d_{2} & -b
\end{array}\right],
\end{aligned}
$$




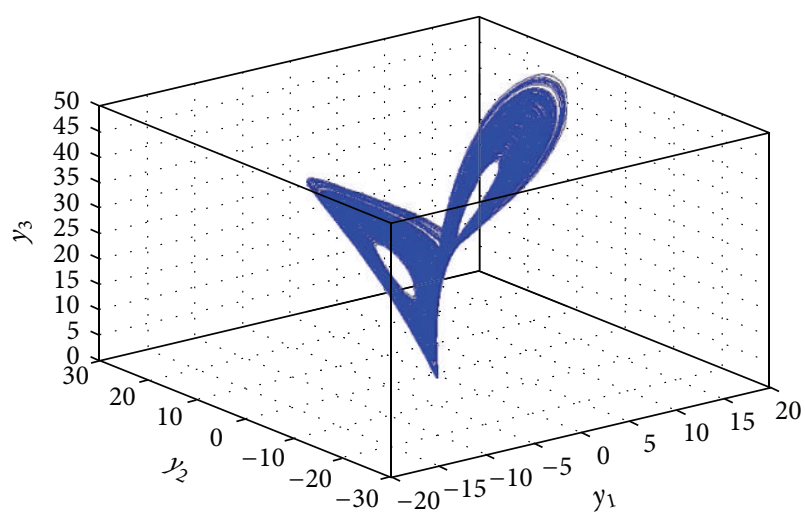

Figure 3: Chaotic behavior of slave fuzzy Lorenz system.

$$
\begin{aligned}
\widetilde{A}_{2} & =\left[\begin{array}{ccc}
-a & a & 0 \\
c & -1 & d_{2} \\
0 & -d_{2} & -b
\end{array}\right], \\
\widetilde{M}_{1}(x) & =\frac{1}{2}\left(1+\frac{y_{1}}{d_{2}}\right), \\
\widetilde{M}_{2}(x) & =\frac{1}{2}\left(1-\frac{y_{1}}{d_{2}}\right), \\
U_{1} & =\left[u_{11}, u_{12}, u_{13}\right]^{T}, \\
U_{2} & =\left[u_{21}, u_{22}, u_{23}\right]^{T}
\end{aligned}
$$

and $d_{2}=20 . \widetilde{M}_{1}$ and $\widetilde{M}_{2}$ are fuzzy set of Lorenz system. Here, we call (37) the first linear subsystem under the fuzzy rule and (38) the second linear subsystem under the fuzzy rule. As a result, $U_{1}$ and $U_{2}$ are controllers of the first and second subsystems. The final output of the fuzzy Lorenz system is inferred as follows and the chaotic behavior without using controllers is shown in Figure 3

$$
\dot{Y}(t)=\sum_{i=1}^{2} \widetilde{H}_{i}\left(\widetilde{A}_{i} Y(t)+U_{i}\right)
$$

where

$$
\begin{aligned}
\widetilde{H}_{1} & =\frac{\widetilde{M}_{1}}{\widetilde{M}_{1}+\widetilde{M}_{2}}, \\
\widetilde{H}_{2} & =\frac{\widetilde{M}_{2}}{\widetilde{M}_{1}+\widetilde{M}_{2}} .
\end{aligned}
$$

We further summarize all the parameters in Section 3.2 into Table 2.

3.3. Fuzzy Modeling of Chen System as Slave System. For Chen system as slave system

$$
\begin{aligned}
& \dot{z}_{1}=e\left(z_{2}-z_{1}\right)+u_{1}, \\
& \dot{z}_{2}=(g-e) z_{1}-z_{1} z_{3}+g z_{2}+u_{2}, \\
& \dot{z}_{3}=z_{1} z_{2}-f z_{3}+u_{3}
\end{aligned}
$$

TABLE 2

\begin{tabular}{lcc}
\hline System & Parameters & Initial states \\
\hline Fuzzy Lorenz & $a=10, b=8 / 3$, & $\left(y_{1}, y_{2}, y_{3}\right)=(0.6,3,10)$ \\
system (slave) & $c=28, d_{2}=20$ & \\
\hline
\end{tabular}

where $e, f, g$ are the parameters. When $e=35, f=3$, $g=28$, and initial states are $(0.5,1,5)$, the dynamic behavior is chaotic. Assume that $z_{1} \in\left[-d_{3}, d_{3}\right]$ and $d_{3}>0$; then Chen system can be exactly represented by T-S fuzzy model as follows:

Rule 1: IF $z$ is $N_{1}$, THEN $\dot{Z}(t)=B_{1} Z(t)$,

Rule 2: IF $z$ is $N_{2}$, THEN $\dot{Z}(t)=B_{2} Z(t)$.

Here

$$
\begin{aligned}
Z & =\left[z_{1}, z_{2}, z_{3}\right]^{T}, \\
B_{1} & =\left[\begin{array}{ccc}
-e & e & 0 \\
(g-e) & g & -d_{3} \\
0 & d_{3} & -f
\end{array}\right], \\
B_{2} & =\left[\begin{array}{ccc}
-e & e & 0 \\
(g-e) & g & d_{3} \\
0 & -d_{3} & -f
\end{array}\right], \\
N_{1}(z) & =\frac{1}{2}\left(1+\frac{z_{1}}{d_{3}}\right), \\
N_{2}(z) & =\frac{1}{2}\left(1-\frac{z_{1}}{d_{3}}\right) \\
U_{1} & =\left[u_{11}, u_{12}, u_{13}\right]^{T} \\
U_{2} & =\left[u_{21}, u_{22}, u_{23}\right]^{T}
\end{aligned}
$$

and $d_{3}=30 . N_{1}$ and $N_{2}$ are fuzzy set of Lorenz system. Here, we call (43) the first liner subsystem under the fuzzy rule and (44) the second liner subsystem under the fuzzy rule. As a result, $U_{1}$ and $U_{2}$ are controllers of the first and second subsystems. The final output of the fuzzy Chen system is inferred as follows and the chaotic behavior without using controllers is shown in Figure 4

$$
\dot{Z}(t)=\sum_{i=1}^{2} I_{i}\left(B_{i} Z(t)+U_{i}\right)
$$

where

$$
\begin{aligned}
& I_{1}=\frac{N_{1}}{N_{1}+N_{2}}, \\
& I_{2}=\frac{N_{2}}{N_{1}+N_{2}} .
\end{aligned}
$$


TABLE 3

\begin{tabular}{lcc}
\hline System & Parameters & Initial states \\
\hline Fuzzy Chen & $e=35, f=3, g=28$, & $\left(z_{1}, z_{2}, z_{3}\right)=(0.5,1,5)$ \\
system (slave) & $d_{3}=30$ & \\
\hline
\end{tabular}

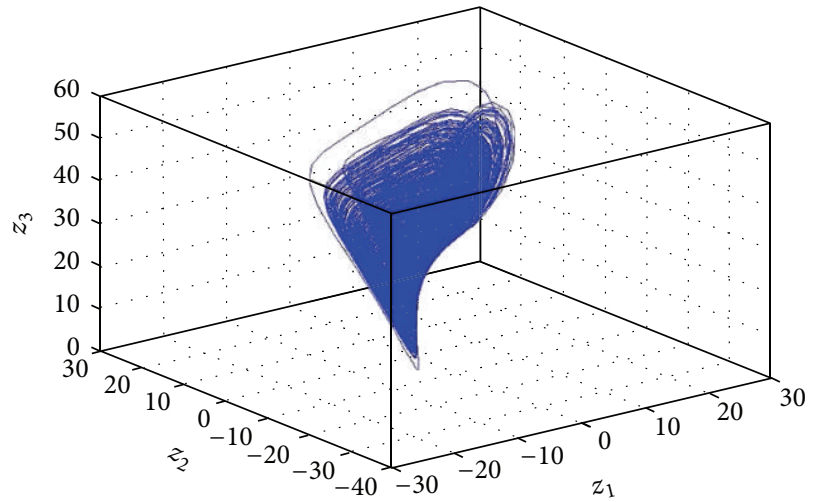

FIgURE 4: Chaotic behavior of slave fuzzy Chen system.

We further summarize all the parameters in Section 3.3 into Table 3 .

3.4. Fuzzy Modeling of Rossler's System as Functional System. For Rossler's system as functional system

$$
\begin{aligned}
& \dot{w}_{1}=-w_{2}-w_{3}, \\
& \dot{w}_{2}=w_{1}+h w_{2}, \\
& \dot{w}_{3}=i+w_{1} w_{3}-j w_{3},
\end{aligned}
$$

where $e, f, g$ are the parameters. When $h=0.2, i=0.2, j=$ 5.7, and initial states are $(10,15,11)$, the dynamic behavior is chaotic. Assume that $w_{1} \in\left[-d_{4}, d_{4}\right]$ and $d_{4}>0$; then Chen system can be exactly represented by T-S fuzzy model as follows:

Rule 1: IF $w$ is $O_{1}$, THEN $\dot{W}(t)=C_{1} W(t)+c_{1}$,

Rule 2: IF $w$ is $O_{2}$, THEN $\dot{W}(t)=C_{2} W(t)+c_{2}$.

Here

$$
\begin{gathered}
W=\left[w_{1}, w_{2}, w_{3}\right]^{T}, \\
C_{1}=\left[\begin{array}{ccc}
0 & -1 & -1 \\
1 & h & 0 \\
0 & d_{4} & -j
\end{array}\right],
\end{gathered}
$$

TABLE 4

\begin{tabular}{lcc}
\hline System & Parameters & Initial states \\
\hline $\begin{array}{l}\text { Fuzzy Rossler's } \\
\text { system }\end{array}$ & $h=0.2, i=0.2$, & \\
(functional & $j=5.7, d_{4}=10$ & $\left(w_{1}, w_{2}, w_{3}\right)=(10,15,11)$ \\
system) & & \\
\hline
\end{tabular}

$$
\begin{aligned}
& C_{2}=\left[\begin{array}{ccc}
0 & -1 & -1 \\
1 & h & 0 \\
0 & -d_{4} & -j
\end{array}\right], \\
& c_{1}=\left[\begin{array}{l}
0 \\
0 \\
i
\end{array}\right], \\
& c_{2}=\left[\begin{array}{l}
0 \\
0 \\
i
\end{array}\right]
\end{aligned}
$$

$$
\begin{aligned}
& O_{1}(z)=\frac{1}{2}\left(1+\frac{w_{1}}{d_{4}}\right), \\
& O_{2}(z)=\frac{1}{2}\left(1-\frac{w_{1}}{d_{4}}\right),
\end{aligned}
$$

and $d_{4}=10 . O_{1}$ and $O_{2}$ are fuzzy set of Lorenz system. Here, we call (49) the first liner subsystem under the fuzzy rule and (50) the second liner subsystem under the fuzzy rule. The final output of the fuzzy Rossler's system is inferred as follows and the chaotic behavior is shown in Figure 5

$$
\dot{Z}(t)=\sum_{i=1}^{2} J_{i}\left(C_{i} Z(t)+c_{i}\right)
$$

where

$$
\begin{aligned}
& J_{1}=\frac{O_{1}}{O_{1}+O_{2}}, \\
& J_{2}=\frac{O_{2}}{O_{1}+O_{2}} .
\end{aligned}
$$

We further summarize all the parameters in Section 3.4 into Table 4.

\section{Numerical Simulation Results of the Bioinspired Strategy}

In this section, there are three main CASEs for illustrations, Case 1: the generalized synchronization error function is $e_{i}=\left(x_{i}-y_{i}+100\right),(i=1,2,3)$, Case 2 : the generalized synchronization error function is $e_{i}=\left(x_{i}-z_{i}+100\right),(i=$ $1,2,3)$, and Case 3: the generalized synchronization error function is $e_{i}=x_{i}-z_{i}+w_{i}+100,(i=1,2,3)$. A set of 


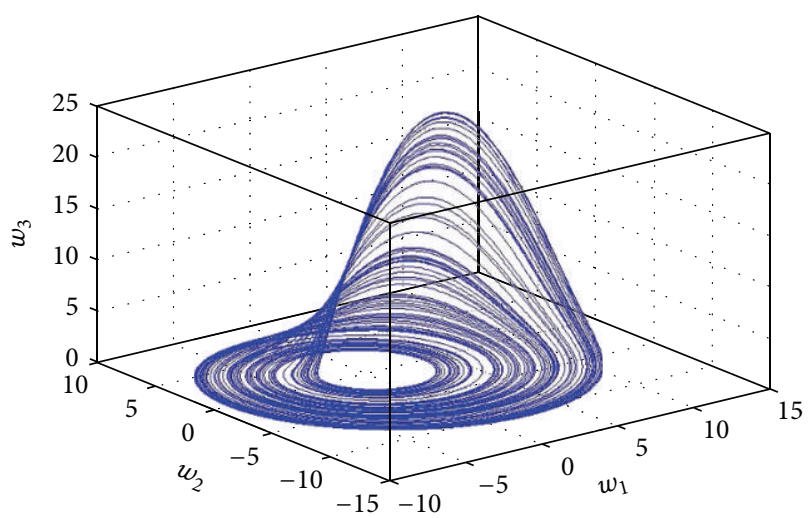

Figure 5: Chaotic behavior of fuzzy Rossler's system as functional system. simple-form and efficient Lyapunov function based on the natural Bioinspired stability theory is directly used to achieve generalized synchronization of two fuzzy chaotic systems.

Case 1. The generalized synchronization error function is $e_{i}=$ $\left(x_{i}-y_{i}+100\right),(i=1,2,3)$.

The addition of 100 makes the error dynamics always happen in first quadrant. Our goal is $y_{i}=x_{i}+100$; that is,

$$
\lim _{t \rightarrow \infty} e_{i}=\lim _{t \rightarrow \infty}\left(x_{i}-y_{i}+100\right)=0 \quad(i=1,2,3) .
$$

$$
\begin{aligned}
\{e\} & =\left[\begin{array}{l}
e_{1} \\
e_{2} \\
e_{3}
\end{array}\right]=\left[\begin{array}{l}
x_{1}-y_{1}+100 \\
x_{2}-y_{2}+100 \\
x_{3}-y_{3}+100
\end{array}\right], \\
\{\dot{e}\} & =\left[\begin{array}{l}
\dot{e}_{1} \\
\dot{e}_{2} \\
\dot{e}_{3}
\end{array}\right]=\left[\begin{array}{l}
\dot{x}_{1}-\dot{y}_{1} \\
\dot{x}_{2}-\dot{y}_{2} \\
\dot{x}_{3}-\dot{y}_{3}
\end{array}\right]=\sum_{i=1}^{2} H_{i} A_{i} X(t)-\sum_{i=1}^{2} \widetilde{H}_{i}\left(\widetilde{A}_{i} Y(t)+U_{i}\right) \\
& =\left[\begin{array}{c}
H_{1} a\left(x_{2}-x_{1}\right)-\widetilde{H}_{1}\left(a\left(y_{2}-y_{1}\right)+u_{11}\right)+H_{2} a\left(x_{2}-x_{1}\right)-\widetilde{H}_{2}\left(a\left(y_{2}-y_{1}\right)+u_{21}\right) \\
H_{1}\left(c x_{1}-d_{1} x_{3}-x_{2}\right)-\widetilde{H}_{1}\left(c y_{1}-d_{2} y_{3}-y_{2}+u_{12}\right)+H_{2}\left(c x_{1}+d_{1} x_{3}-x_{2}\right)-\widetilde{H}_{2}\left(c y_{1}+d_{2} y_{3}-y_{2}+u_{22}\right)
\end{array}\right] .
\end{aligned}
$$

Before control action, the error dynamics always happens in first quadrant as shown in Figure 6. By the natural bioinspired stability theory, one can choose a Lyapunov function in the form of a positive definite function in first quadrant:

$$
V=e_{1}+e_{2}+e_{3}>0 .
$$

Its time derivative through error dynamics (55) is

$$
\begin{aligned}
\dot{V} & =\dot{e}_{1}+\dot{e}_{2}+\dot{e}_{3}=\left(H_{1} a\left(x_{2}-x_{1}\right)\right. \\
& -\widetilde{H}_{1}\left(a\left(y_{2}-y_{1}\right)+u_{11}\right)+H_{2} a\left(x_{2}-x_{1}\right) \\
& \left.-\widetilde{H}_{2}\left(a\left(y_{2}-y_{1}\right)+u_{21}\right)\right) \\
& +\left(H_{1}\left(c x_{1}-d_{1} x_{3}-x_{2}\right)\right. \\
& -\widetilde{H}_{1}\left(c y_{1}-d_{2} y_{3}-y_{2}+u_{12}\right) \\
& +H_{2}\left(c x_{1}+d_{1} x_{3}-x_{2}\right) \\
& \left.-\widetilde{H}_{2}\left(c y_{1}+d_{2} y_{3}-y_{2}+u_{22}\right)\right)+\left(H_{1}\left(d_{1} x_{2}-b x_{3}\right)\right. \\
& -\widetilde{H}_{1}\left(\left(d_{2} y_{2}-b y_{3}\right)+u_{13}\right)+H_{2}\left(-d_{1} x_{2}-b x_{3}\right) \\
& \left.-\widetilde{H}_{2}\left(\left(-d_{2} y_{2}-b y_{3}\right)+u_{23}\right)\right) .
\end{aligned}
$$

Choose

$$
\begin{aligned}
& u_{11}=\frac{H_{1}}{\widetilde{H}_{1}} a\left(x_{2}-x_{1}\right)-a\left(y_{2}-y_{1}\right)+e_{1}, \\
& u_{12}=\frac{H_{1}}{\widetilde{H}_{1}}\left(-d_{1} x_{3}+c x_{2}\right)-\left(-d_{2} y_{3}+c y_{2}\right)+e_{2}, \\
& u_{13}=\frac{H_{1}}{\widetilde{H}_{1}}\left(d_{1} x_{2}-b x_{3}\right)-\left(d_{2} y_{2}-b y_{3}\right)+e_{3}, \\
& u_{21}=\frac{H_{2}}{\widetilde{H}_{2}} a\left(x_{2}-x_{1}\right)-a\left(y_{2}-y_{1}\right)+e_{1}, \\
& u_{22}=\frac{H_{2}}{\widetilde{H}_{2}}\left(d_{1} x_{3}+c x_{2}\right)-\left(d_{2} y_{3}+c y_{2}\right)+e_{2}, \\
& u_{23}=\frac{H_{2}}{\widetilde{H}_{2}}\left(-d_{1} x_{2}-b x_{3}\right)-\left(-d_{2} y_{2}-b y_{3}\right)+e_{3} .
\end{aligned}
$$

We obtain

$$
\dot{V}=-e_{1}-e_{2}-e_{3}<0,
$$

which is negative definite function in the first quadrant. The numerical results are shown in Figures 7 and 8 . 


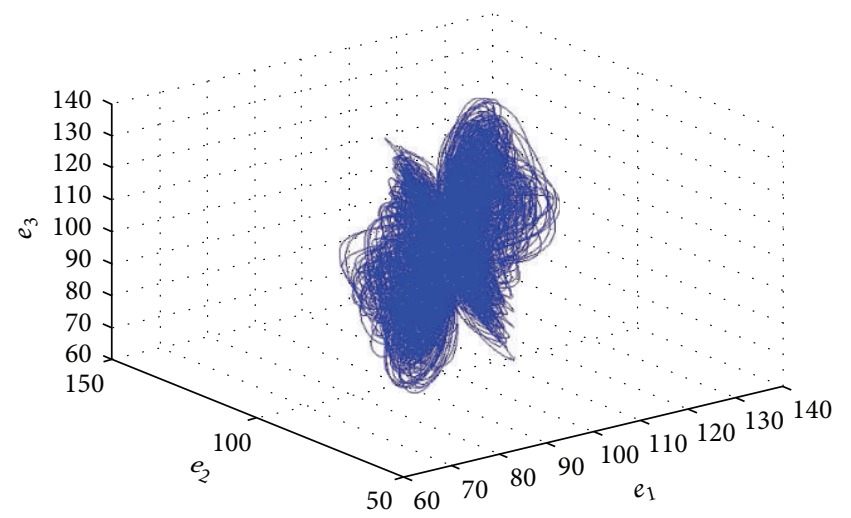

Figure 6: Phase portraits of error dynamics for Case 1.

In Figures 7 and 8, the master and slave systems are two identical Lorenz systems with different initial conditions, where the parameters $a=10, b=8 / 3, c=28, d_{1}=30, d_{2}=$ 20 , initial states are $\left(x_{1}, x_{2}, x_{3}\right)=(0.5,1,5)$ for master system, and $\left(y_{1}, y_{2}, y_{3}\right)=(0.6,3,10)$ for slave system. The control procedure is arranged that, in the initial $30 \mathrm{sec}$, the system is in a nature situation without any control. The designed controllers operate after $30 \mathrm{sec}$ to show the high efficiency of our bioinspired controllers. In Figure 7, it is clear that the error states $e_{1}, e_{2}, e_{3}$ almost immediately smoothly approach zero after the controllers put in at $30 \mathrm{sec}$. Also, in Figure 8, the states of the slave system are all synchronized to the states of the target system with high performance.

Case 2. The generalized synchronization error function is $e_{i}=\left(x_{i}-z_{i}+100\right),(i=1,2,3)$.

The addition of 100 makes the error dynamics always happen in first quadrant. Our goal is $z_{i}=x_{i}+100$; that is,

$$
\lim _{t \rightarrow \infty} e_{i}=\lim _{t \rightarrow \infty}\left(x_{i}-z_{i}+100\right)=0 \quad(i=1,2,3) .
$$

The error and error dynamics are $\{e\}=\left[\begin{array}{l}e_{1} \\ e_{2} \\ e_{3}\end{array}\right]=\left[\begin{array}{l}x_{1}-z_{1}+100 \\ x_{2}-z_{2}+100 \\ x_{3}-z_{3}+100\end{array}\right]$,

$\{\dot{e}\}$

$$
\begin{aligned}
& =\left[\begin{array}{l}
\dot{e}_{1} \\
\dot{e}_{2} \\
\dot{e}_{3}
\end{array}\right]=\left[\begin{array}{l}
\dot{x}_{1}-\dot{z}_{1} \\
\dot{x}_{2}-\dot{z}_{2} \\
\dot{x}_{3}-\dot{z}_{3}
\end{array}\right]=\sum_{i=1}^{2} H_{i} A_{i} X(t)-\sum_{i=1}^{2} I_{i}\left(B_{i} Z(t)+U_{i}\right) \\
& =\left[\begin{array}{r}
H_{1}\left(a\left(x_{2}-x_{1}\right)\right)-I_{1}\left(e\left(z_{2}-z_{1}\right)+u_{11}\right)+H_{2}\left(a\left(x_{2}-x_{1}\right)\right)-I_{2}\left(e\left(z_{2}-z_{1}\right)+u_{21}\right) \\
H_{1}\left(c x_{1}-d_{1} x_{3}-x_{2}\right)-I_{1}\left((g-e) z_{1}-d_{3} z_{3}+g z_{2}+u_{12}\right)+H_{2}\left(c x_{1}+d_{1} x_{3}-x_{2}\right)-I_{2}\left((g-e) z_{1}+d_{3} z_{3}+g z_{2}+u_{22}\right) \\
H_{1}\left(d_{1} x_{2}-b x_{3}\right)-I_{1}\left(d_{3} z_{2}-f z_{3}+u_{13}\right)+H_{2}\left(-d_{1} x_{2}-b x_{3}\right)-I_{2}\left(-d_{3} z_{2}-f z_{3}+u_{23}\right)
\end{array}\right] .
\end{aligned}
$$

Before control action, the error dynamics always happens in first quadrant as shown in Figure 9. By the natural bioinspired stability theory, one can choose a Lyapunov function in the form of a positive definite function in first quadrant:

$$
V=e_{1}+e_{2}+e_{3}>0 \text {. }
$$

Its time derivative through error dynamics (61) is

$$
\begin{aligned}
\dot{V} & =\dot{e}_{1}+\dot{e}_{2}+\dot{e}_{3}=\left(H_{1}\left(a\left(x_{2}-x_{1}\right)\right)\right. \\
& -I_{1}\left(e\left(z_{2}-z_{1}\right)+u_{11}\right)+H_{2}\left(a\left(x_{2}-x_{1}\right)\right) \\
& \left.-I_{2}\left(e\left(z_{2}-z_{1}\right)+u_{21}\right)\right)+\left(H_{1}\left(c x_{1}-d_{1} x_{3}-x_{2}\right)\right. \\
& -I_{1}\left((g-e) z_{1}-d_{3} z_{3}+g z_{2}+u_{12}\right) \\
& +H_{2}\left(c x_{1}+d_{1} x_{3}-x_{2}\right) \\
& \left.-I_{2}\left((g-e) z_{1}+d_{3} z_{3}+g z_{2}+u_{22}\right)\right)
\end{aligned}
$$

$$
\begin{aligned}
& +\left(H_{1}\left(d_{1} x_{2}-b x_{3}\right)-I_{1}\left(d_{3} z_{2}-f z_{3}+u_{13}\right)\right. \\
& \left.+H_{2}\left(-d_{1} x_{2}-b x_{3}\right)-I_{2}\left(-d_{3} z_{2}-f z_{3}+u_{23}\right)\right) .
\end{aligned}
$$

Choose

$$
\begin{aligned}
u_{11}= & \frac{H_{1}}{I_{1}}\left(a\left(x_{2}-x_{1}\right)\right)-\left(e\left(z_{2}-z_{1}\right)\right)+e_{1}, \\
u_{12}= & \frac{H_{1}}{I_{1}}\left(c x_{1}-d_{1} x_{3}-x_{2}\right) \\
& -\left((g-e) z_{1}-d_{3} z_{3}+g z_{2}\right)+e_{2}, \\
u_{13}= & \frac{H_{1}}{I_{1}}\left(d_{1} x_{2}-b x_{3}\right)-\left(d_{3} z_{2}-f z_{3}\right)+e_{3}, \\
u_{21}= & \frac{H_{2}}{I_{2}}\left(a\left(x_{2}-x_{1}\right)\right)-\left(e\left(z_{2}-z_{1}\right)\right)+e_{1}, \\
u_{22}= & \frac{H_{2}}{I_{2}}\left(c x_{1}+d_{1} x_{3}-x_{2}\right)
\end{aligned}
$$



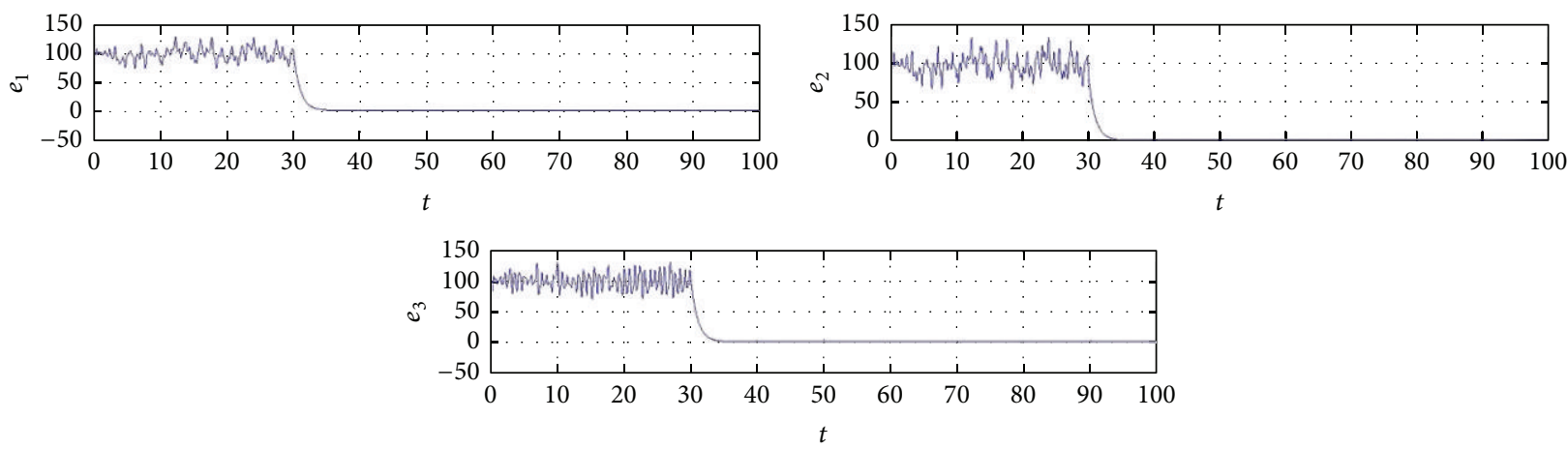

Figure 7: Time histories of error dynamics for Case 1 -the error states $e_{1}, e_{2}, e_{3}$ almost immediately smoothly approach zero after the controllers put in at $30 \mathrm{sec}$.
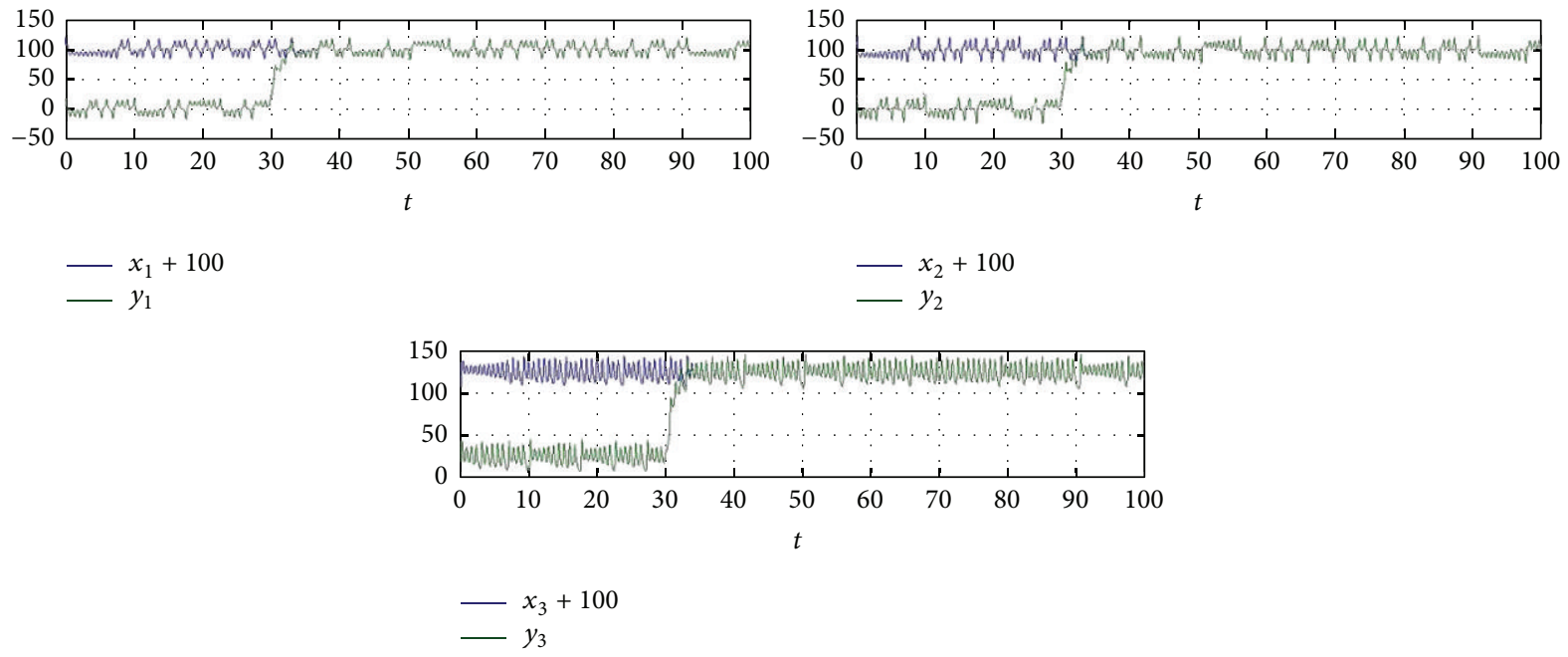

FIgURE 8: Time histories of $x_{1}, x_{2}, x_{3}$ and $y_{1}, y_{2}, y_{3}$ for Case 1-the states of the slave system are all synchronized to the states of the target system with high performance.

$$
\begin{gathered}
-\left((g-e) z_{1}+d_{3} z_{3}+g z_{2}\right)+e_{2} \\
u_{23}=\frac{H_{2}}{I_{2}}\left(-d_{1} x_{2}-b x_{3}\right)-\left(-d_{3} z_{2}-f z_{3}\right)+e_{3} .
\end{gathered}
$$

We obtain

$$
\dot{V}=-e_{1}^{2}-e_{2}^{2}-e_{3}^{2}<0,
$$

which is negative definite function in the first quadrant. The numerical results are shown in Figures 10 and 11. In Figures 10 and 11 , Lorenz system with initial state $\left(x_{1}, x_{2}, x_{3}\right)=(0.5,1,5)$ in (34) is considered as master system and Chen system with initial state $\left(z_{1}, z_{2}, z_{3}\right)=(0.5,1,5)$ in $(46)$ is referring to the slave system, where the parameters $a=10, b=8 / 3, c=28$, $e=35, f=3, g=28, d_{1}=30, d_{3}=30$. The control procedure is arranged that, in the initial $30 \mathrm{sec}$, the system is in a nature situation without any control. The designed controllers operate after $30 \mathrm{sec}$ to show the high efficiency of our bioinspired controllers. In Figure 10, it is clear that

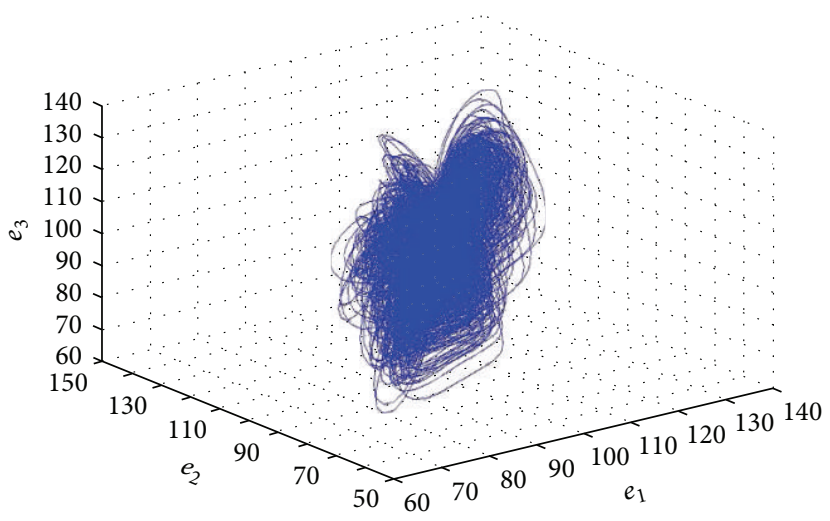

Figure 9: Phase portraits of error dynamics for Case 2.

the error states $e_{1}, e_{2}, e_{3}$ almost immediately smoothly approach zero after the controllers put in at $30 \mathrm{sec}$. Also, in Figure 11, the states of the slave system are all synchronized to the states of the target system with high performance. 

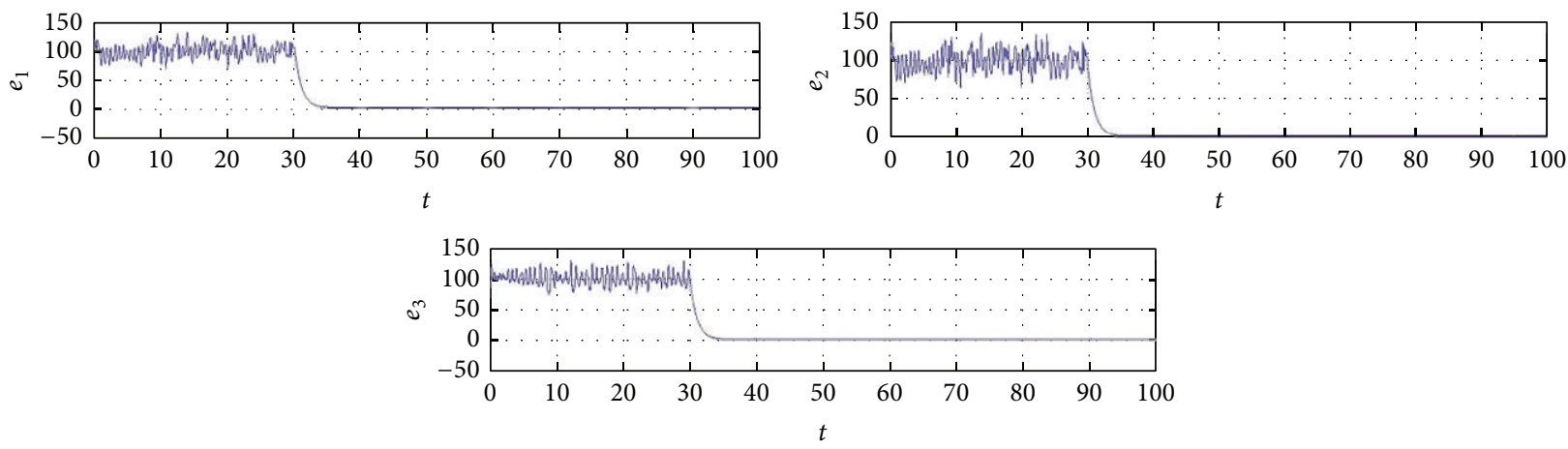

Figure 10: Time histories of error dynamics for Case 2-the error states $e_{1}, e_{2}, e_{3}$ almost immediately smoothly approach zero after the controllers put in at $30 \mathrm{sec}$.
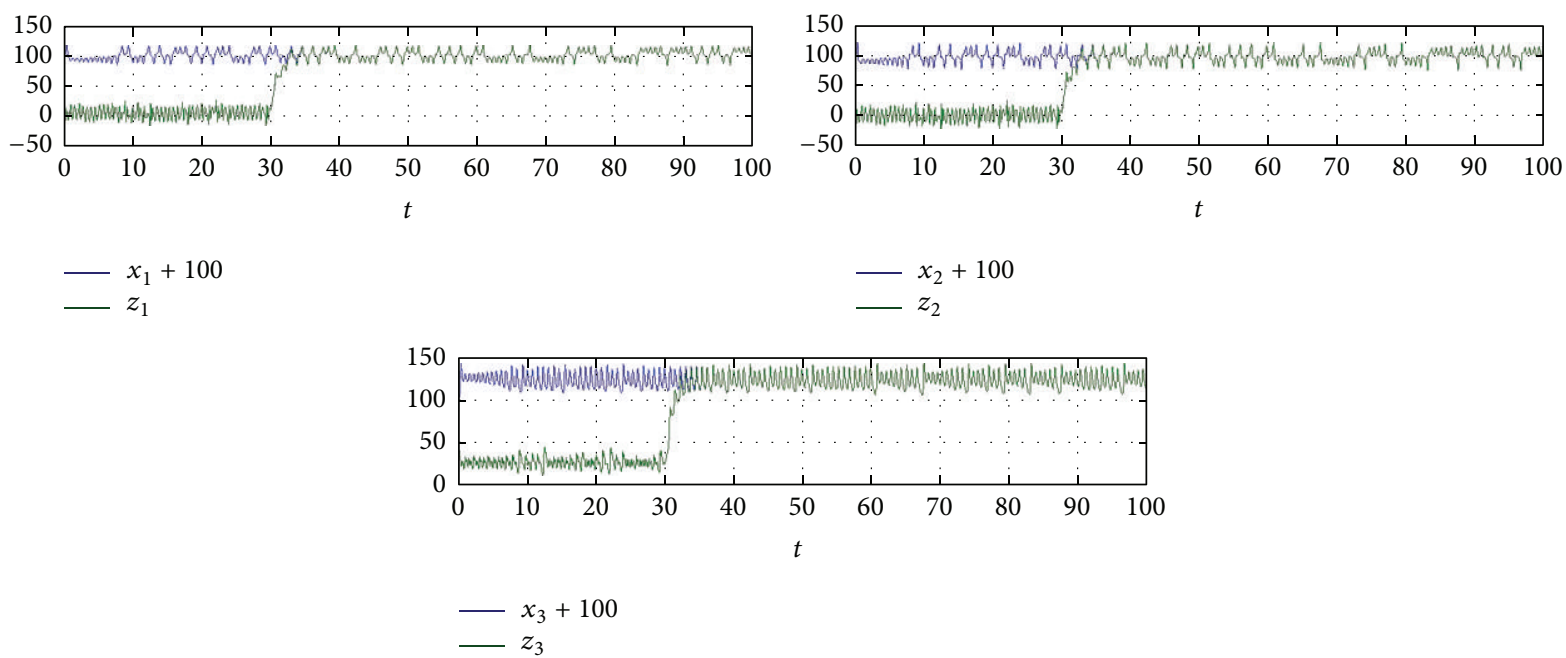

Figure 11: Time histories of $x_{1}+100, x_{2}+100, x_{3}+100$, and $z_{1}, z_{2}, z_{3}$ for Case 2 -the states of the slave system are all synchronized to the states of the target system with high performance.

Case 3. The generalized synchronization error function is $e_{i}=x_{i}-z_{i}+w_{i}+100,(i=1,2,3)$, where the states $\left(z_{1}, z_{2}, z_{3}\right)$ of the slave Chen system in (46) are going to achieve generalized synchronization of a complicated designed goal system - the states $\left(x_{1}, x_{2}, x_{3}\right)$ of Lorenz system in (32) with the states $\left(w_{1}, w_{2}, w_{3}\right)$ of the functional system in (52).

$\{e\}=\left[\begin{array}{l}e_{1} \\ e_{2} \\ e_{3}\end{array}\right]=\left[\begin{array}{l}x_{1}-z_{1}+w_{1}+100 \\ x_{2}-z_{2}+w_{2}+100 \\ x_{3}-z_{3}+w_{3}+100\end{array}\right]$,

$\{\dot{e}\}$

$$
\begin{aligned}
& =\left[\begin{array}{c}
\dot{e}_{1} \\
\dot{e}_{2} \\
\dot{e}_{3}
\end{array}\right]=\left[\begin{array}{c}
\dot{x}_{1}-\dot{w}_{1} \\
\dot{x}_{2}-\dot{w}_{2} \\
\dot{x}_{3}-\dot{w}_{3}
\end{array}\right]=\sum_{i=1}^{2} H_{i} A_{i} X(t)+\sum_{i=1}^{2} J_{i}\left(C_{i} Z(t)+c_{i}\right)-\sum_{i=1}^{2} I_{i}\left(B_{i} Z(t)+U_{i}\right) \\
& =\left[\begin{array}{r}
H_{1}\left(a\left(x_{2}-x_{1}\right)\right)+J_{1}\left(-w_{2}-w_{3}\right)-I_{1}\left(e\left(z_{2}-z_{1}\right)+u_{11}\right)+H_{2}\left(a\left(x_{2}-x_{1}\right)\right)+J_{2}\left(-w_{2}-w_{3}\right)-I_{2}\left(e\left(z_{2}-z_{1}\right)+u_{21}\right) \\
H_{1}\left(c x_{1}-d_{1} x_{3}-x_{2}\right)+J_{1}\left(w_{1}+h w_{2}\right)-I_{1}\left((g-e) z_{1}-d_{3} z_{3}+g z_{2}+u_{12}\right)+H_{2}\left(c x_{1}+d_{1} x_{3}-x_{2}\right)+J_{2}\left(w_{1}+h w_{2}\right)-I_{2}\left((g-e) z_{1}+d_{3} z_{3}+g z_{2}+u_{22}\right) \\
H_{1}\left(d_{1} x_{2}-b x_{3}\right)+J_{1}\left(i+d_{4} w_{3}-j w_{3}\right)-I_{1}\left(d_{3} z_{2}-f z_{3}+u_{13}\right)+H_{2}\left(-d_{1} x_{2}-b x_{3}\right)+J_{2}\left(i-d_{4} w_{3}-j w_{3}\right)-I_{2}\left(-d_{3} z_{2}-f z_{3}+u_{23}\right)
\end{array}\right] .
\end{aligned}
$$


Before control action, the error dynamics always happens in first quadrant as shown in Figure 12. By the natural bioinspired stability theory, one can choose a Lyapunov function in the form of a positive definite function in first quadrant:

$$
V=e_{1}+e_{2}+e_{3}>0 .
$$

Its time derivative through error dynamics (67) is

$$
\begin{aligned}
\dot{V} & =\dot{e}_{1}+\dot{e}_{2}+\dot{e}_{3}=\left(H_{1}\left(a\left(x_{2}-x_{1}\right)\right)+J_{1}\left(-w_{2}-w_{3}\right)\right. \\
& -I_{1}\left(e\left(z_{2}-z_{1}\right)+u_{11}\right)+H_{2}\left(a\left(x_{2}-x_{1}\right)\right) \\
& \left.+J_{2}\left(-w_{2}-w_{3}\right)-I_{2}\left(e\left(z_{2}-z_{1}\right)+u_{21}\right)\right) \\
& +\left(H_{1}\left(c x_{1}-d_{1} x_{3}-x_{2}\right)+J_{1}\left(w_{1}+h w_{2}\right)\right. \\
& -I_{1}\left((g-e) z_{1}-d_{3} z_{3}+g z_{2}+u_{12}\right) \\
& +H_{2}\left(c x_{1}+d_{1} x_{3}-x_{2}\right)+J_{2}\left(w_{1}+h w_{2}\right) \\
& \left.-I_{2}\left((g-e) z_{1}+d_{3} z_{3}+g z_{2}+u_{22}\right)\right) \\
& +\left(H_{1}\left(d_{1} x_{2}-b x_{3}\right)+J_{1}\left(i+d_{4} w_{3}-j w_{3}\right)\right. \\
& -I_{1}\left(d_{3} z_{2}-f z_{3}+u_{13}\right)+H_{2}\left(-d_{1} x_{2}-b x_{3}\right) \\
& \left.+J_{2}\left(i-d_{4} w_{3}-j w_{3}\right)-I_{2}\left(-d_{3} z_{2}-f z_{3}+u_{23}\right)\right) .
\end{aligned}
$$

Choose

$$
\begin{aligned}
u_{11}= & \frac{H_{1}}{I_{1}} a\left(x_{2}-x_{1}\right)+\frac{J_{1}}{I_{1}}\left(-w_{2}-w_{3}\right)-e\left(z_{2}-z_{1}\right) \\
& +e_{1}, \\
u_{12}= & \frac{H_{1}}{I_{1}}\left(c x_{1}-d_{1} x_{3}-x_{2}\right)+\frac{J_{1}}{I_{1}}\left(w_{1}+h w_{2}\right) \\
& -\left((g-e) z_{1}-d_{3} z_{3}+g z_{2}\right)+e_{2}, \\
u_{13}= & \frac{H_{1}}{I_{1}}\left(d_{1} x_{2}-b x_{3}\right)+\frac{J_{1}}{I_{1}}\left(i+d_{4} w_{3}-j w_{3}\right) \\
& -\left(d_{3} z_{2}-f z_{3}\right)+e_{3}, \\
u_{21}= & \frac{H_{2}}{I_{2}} a\left(x_{2}-x_{1}\right)+\frac{J_{2}}{I_{2}}\left(-w_{2}-w_{3}\right)-e\left(z_{2}-z_{1}\right) \\
& +e_{1}, \\
u_{22}= & \frac{H_{2}}{I_{2}}\left(c x_{1}+d_{1} x_{3}-x_{2}\right)+\frac{J_{2}}{I_{2}}\left(w_{1}+h w_{2}\right) \\
& -\left((g-e) z_{1}+d_{3} z_{3}+g z_{2}\right)+e_{2}, \\
u_{23}= & \frac{H_{2}}{I_{2}}\left(-d_{1} x_{2}-b x_{3}\right)+\frac{J_{2}}{I_{2}}\left(i-d_{4} w_{3}-j w_{3}\right) \\
& -\left(-d_{3} z_{2}-f z_{3}\right)+e_{3} .
\end{aligned}
$$

We obtain

$$
\dot{V}_{1}=-e_{1}-e_{2}-e_{3}<0,
$$

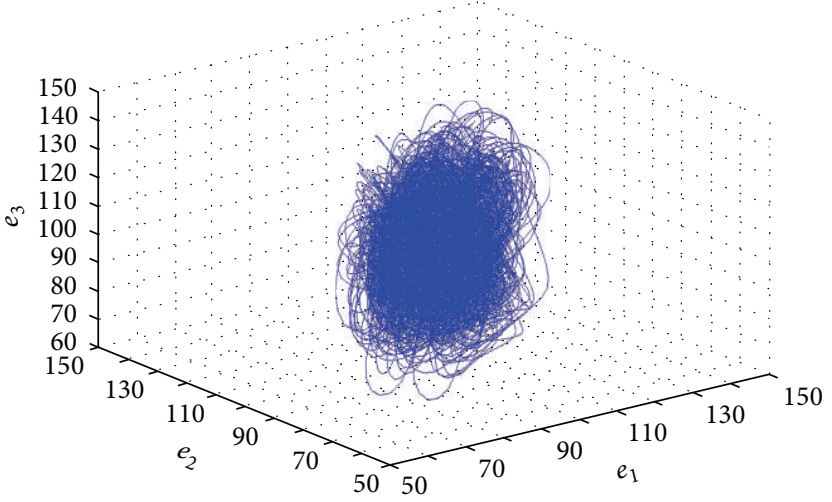

FIgURE 12: Phase portraits of error dynamics for Case 3.

which is negative definite function in the first quadrant. The numerical results are shown in Figures 13 and 14. In Figures 13 and 14, a complicated goal system-Lorenz system with initial state $\left(x_{1}, x_{2}, x_{3}\right)=(0.5,1,5)$ in (34) combined with the functional system with initial state $\left(w_{1}, w_{2}, w_{3}\right)=(10,15,11)$ in (52), is considered as the master system and Chen system with initial state $\left(z_{1}, z_{2}, z_{3}\right)=(0.5,1,5)$ in $(46)$ is referring to the slave system, where the parameters $a=10, b=8 / 3, c=$ 28, $e=35, f=3, g=28, h=0.2, i=0.2, j=5.7, d_{1}=30$, $d_{3}=30, d_{4}=10$. The control procedure is arranged that, in the initial $30 \mathrm{sec}$, the system is in a nature situation without any control. The designed controllers operate after $30 \mathrm{sec}$ to show the high efficiency of our bioinspired controllers. In Figure 12, it is clear that the error states $e_{1}, e_{2}, e_{3}$ almost immediately smoothly approach zero after the controllers put in at $30 \mathrm{sec}$. Also, in Figure 13, the states of the slave system are all synchronized to the states of the target system with high performance.

\section{Conclusions}

In our daily life, there are lots of natural systems whose certain states are always positive, that is, exist in the first quadrant. Consequently, a novel control strategy by using bioinspired stability theory is proposed to achieve generalized fuzzy chaos synchronization. In this paper, a new Lyapunov function based on bioinspired control strategy is proposed to directly achieve chaos synchronization of chaotic systems. Via using this strategy, the new Lyapunov function used is a simple linear homogeneous function of states and the process of synchronization of two fuzzy chaotic systems becomes more simple and the controllers operate after $30 \mathrm{sec}$; the error states $e_{1}, e_{2}, e_{3}$ almost immediately smoothly approach zero. Classical Lorenz, Chen, and Rossler's systems are used in numerical examples and are given to demonstrate the effectiveness of the proposed new strategy. Also, this paper provides a new sight of view for researchers to reference and we are going to further apply the novel bioinspired controlling strategy to other real-world applications in the following stage, such as energy communication system in biological research, virus-immune system, and HIV virusimmune system. 

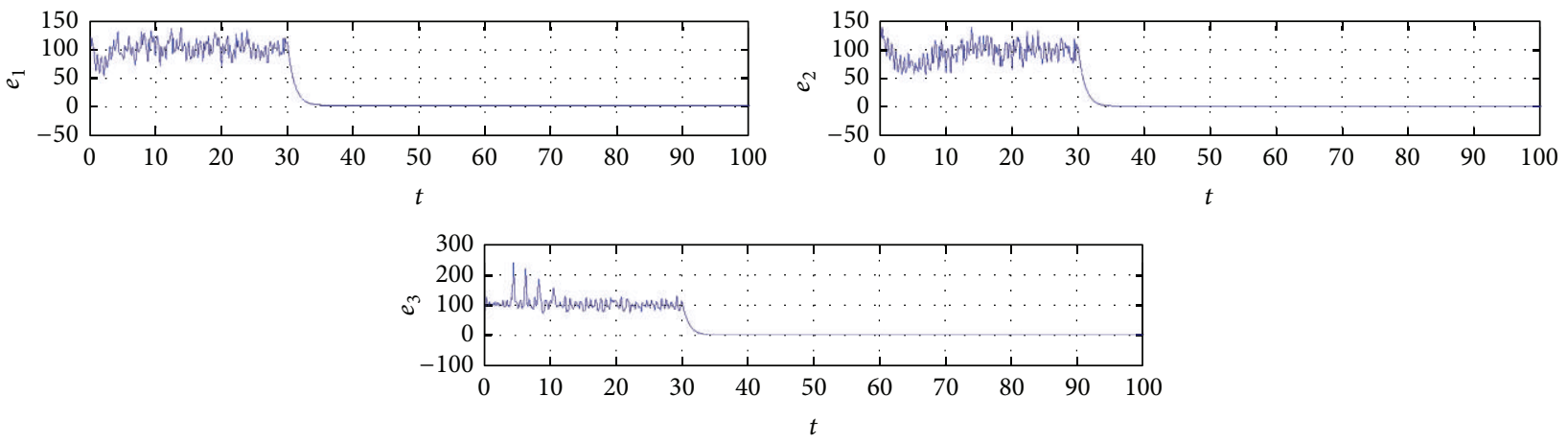

Figure 13: Time histories of error dynamics for Case 3-the error states $e_{1}, e_{2}, e_{3}$ almost immediately smoothly approach zero after the controllers put in at $30 \mathrm{sec}$.
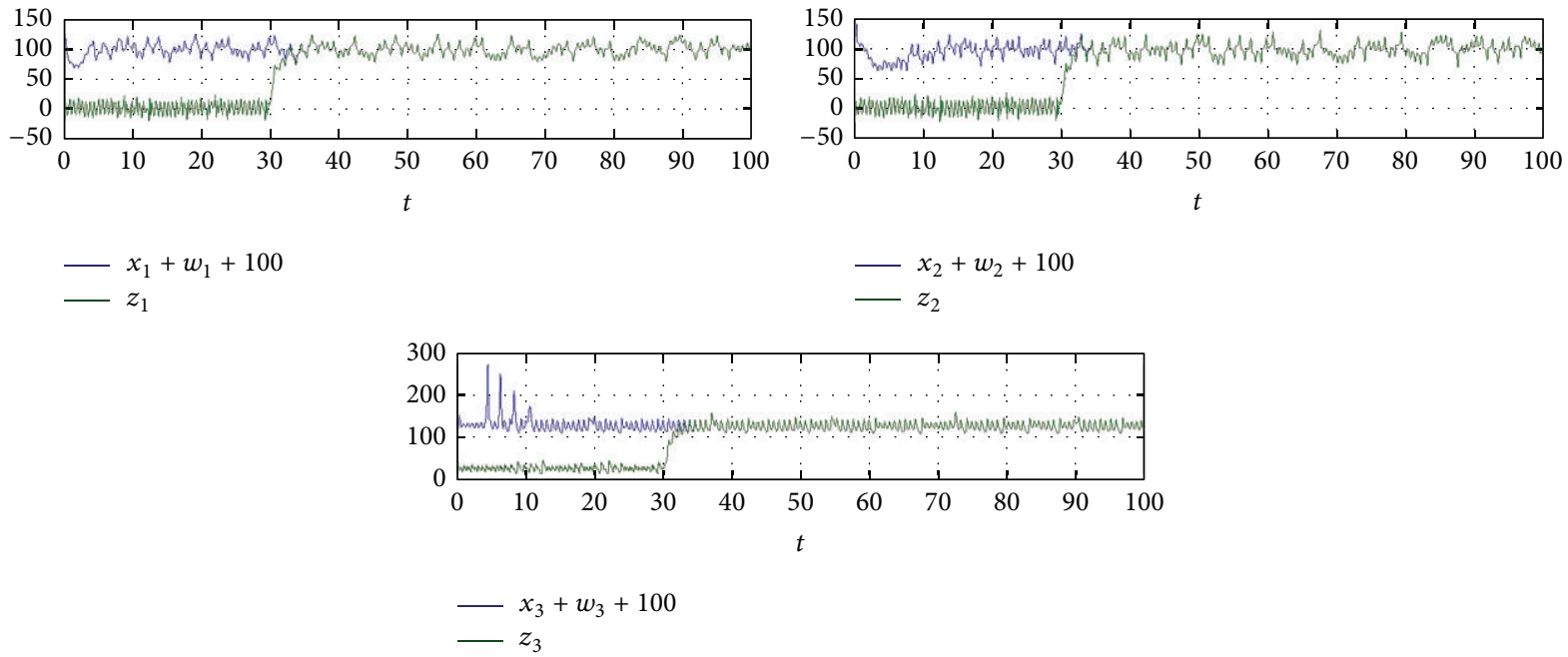

FIGURE 14: Time histories of $x_{1}+w_{1}+100, x_{2}+w_{3}+100, x_{3}+w_{3}+100$, and $z_{1}, z_{2}, z_{3}$ for Case 3 -the states of the slave system are all synchronized to the states of the target system with high performance.

\section{Conflict of Interests}

The authors declare that there is no conflict of interests regarding the publication of this paper.

\section{Acknowledgments}

The authors would like to thank Professor Hsien-Keng Chen for the great assistance and precious advice, especially for the explanations and applications of the GYC Partial Region Stability Theory. Professor Chen is the Professor of the Department of Mechanical Engineering, Hsiuping University of Science and Technology. Also, this work was supported in part by the UST-UCSD International Center of Excellence in Advanced Bio-Engineering sponsored by the Ministry of Science and Technology I-RiCE Program under Grant no. NSC-103-2911-I-009-101. This work was also supported in part by the Aiming for the Top University Plan of National Chiao Tung University, the Ministry of Education, Taiwan, under Contract 104W963, and supported in part by the
Army Research Laboratory and was accomplished under Cooperative Agreement no. W911NF-10-2-0022.

\section{References}

[1] L. M. Pecora and T. L. Carroll, "Synchronization in chaotic systems," Physical Review Letters, vol. 64, no. 8, pp. 821-824, 1990.

[2] A. R. Paul, S. Joshi, A. Jindal, S. P. Maurya, and A. Jain, "Experimental studies of active and passive flow control techniques applied in a twin air-intake," The Scientific World Journal, vol. 2013, Article ID 523759, 8 pages, 2013.

[3] B. Zhu, Y. Chen, and J. Zhao, "Integrated chassis control of active front steering and yaw stability control based on improved inverse nyquist array method," The Scientific World Journal, vol. 2014, Article ID 919847, 14 pages, 2014.

[4] Y. Lan and Q. Li, "Chaos synchronization of a new hyperchaotic system," Applied Mathematics and Computation, vol. 217, no. 5, pp. 2125-2132, 2010. 
[5] S.-Y. Li, C.-H. Yang, C.-T. Lin, L.-W. Ko, and T.-T. Chiu, "Adaptive synchronization of chaotic systems with unknown parameters via new backstepping strategy," Nonlinear Dynamics, vol. 70, no. 3, pp. 2129-2143, 2012.

[6] H. Yang, H. Wang, and H. R. Karimi, "Robust adaptive neural backstepping control for a class of nonlinear systems with dynamic uncertainties," Abstract and Applied Analysis, vol. 2014, Article ID 658671, 12 pages, 2014.

[7] X. Wu, G. Chen, and J. Cai, "Chaos synchronization of the master-slave generalized Lorenz systems via linear state error feedback control," Physica D: Nonlinear Phenomena, vol. 229, no. 1, pp. 52-80, 2007.

[8] X. Yang, K.-X. Peng, and C.-N. Tong, "Robust backstepping control for cold rolling main drive system with nonlinear uncertainties," Abstract and Applied Analysis, vol. 2013, Article ID 387890, 7 pages, 2013.

[9] Z.-M. Ge and S.-Y. Li, "Yang and Yin parameters in the Lorenz system," Nonlinear Dynamics, vol. 62, no. 1-2, pp. 105-117, 2010.

[10] S.-Y. Li and Z.-M. Ge, "Pragmatical adaptive synchronization of different orders chaotic systems with all uncertain parameters via nonlinear control," Nonlinear Dynamics, vol. 64, no. 1-2, pp. 77-87, 2011

[11] S.-Y. Li and Z.-M. Ge, "A novel study of parity and attractor in the time reversed Lorentz system," Physics Letters A, vol. 373, no. 44, pp. 4053-4059, 2009.

[12] M. R. Soltanpour and M. H. Khooban, "A particle swarm optimization approach for fuzzy sliding mode control for tracking the robot manipulator," Nonlinear Dynamics, vol. 74, no. 1-2, pp. 467-478, 2013.

[13] C.-H. Yang, Z.-M. Ge, C.-M. Chang, and S.-Y. Li, "Chaos synchronization and chaos control of quantum-CNN chaotic system by variable structure control and impulse control," Nonlinear Analysis: Real World Applications, vol. 11, no. 3, pp. 1977-1985, 2010.

[14] Y. Shang, Y. Yuan, and F. Yuan, "Adaptive output feedback stabilization of nonholonomic systems with nonlinear parameterization," Abstract and Applied Analysis, vol. 2014, Article ID 623605, 9 pages, 2014.

[15] K. Chakraborty, S. Jana, and T. K. Kar, "Effort dynamics of a delay-induced prey-predator system with reserve," Nonlinear Dynamics, vol. 70, no. 3, pp. 1805-1829, 2012.

[16] S. Jana and T. K. Kar, "A mathematical study of a prey-predator model in relevance to pest control," Nonlinear Dynamics, vol. 74, no. 3, pp. 667-683, 2013.

[17] Z. M. Ge, Y. T. Wong, and S.-Y. Li, “Temporary lag and anticipated synchronization and anti-synchronization of uncoupled time-delayed chaotic systems," Journal of Sound and Vibration, vol. 318, no. 1-2, pp. 267-278, 2008.

[18] S. S. D. Xu, "Super-twisting-algorithm-based terminal sliding mode control for a bioreactor system," Abstract and Applied Analysis, vol. 2014, Article ID 495680, 9 pages, 2014.

[19] Y. G. Zu, Z. H. Zhao, J. H. Yu, and F. J. Yang, Non-Linear Ecological Modelling, Science Press, Beijing, China, 2004.

[20] B. Mukhopadhyay and R. Bhattacharyya, "Analysis of a virusimmune system model with two distinct delays," Nonlinear Studies, vol. 15, no. 1, pp. 37-50, 2008.

[21] A. El-Gohary and A. S. Al-Ruzaiza, "Chaos and adaptive control in two prey, one predator system with nonlinear feedback," Chaos, Solitons \& Fractals, vol. 34, no. 2, pp. 443-453, 2007.

[22] F. A. Khasawneh, D. A. Barton, and B. P. Mann, "Periodic solutions of nonlinear delay differential equations using spectral element method," Nonlinear Dynamics, vol. 67, no. 1, pp. 641658, 2012.

[23] D. Ghosh, "Projective-dual synchronization in delay dynamical systems with time-varying coupling delay," Nonlinear Dynamics, vol. 66, no. 4, pp. 717-730, 2011.

[24] A. C. Chamgoué, R. Yamapi, and P. Woafo, "Bifurcations in a birhythmic biological system with time-delayed noise," Nonlinear Dynamics, vol. 73, no. 4, pp. 2157-2173, 2013.

[25] A. S. Perelson, "Modelling viral and immune system dynamics," Nature Reviews Immunology, vol. 2, no. 1, pp. 28-36, 2002. 


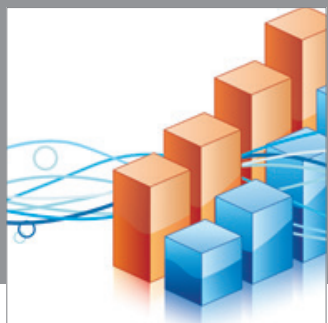

Advances in

Operations Research

mansans

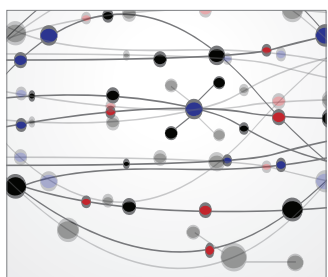

The Scientific World Journal
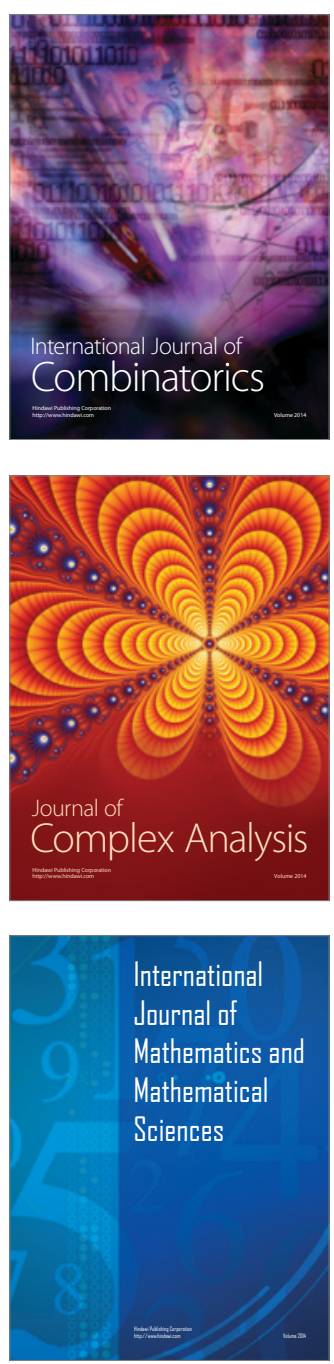
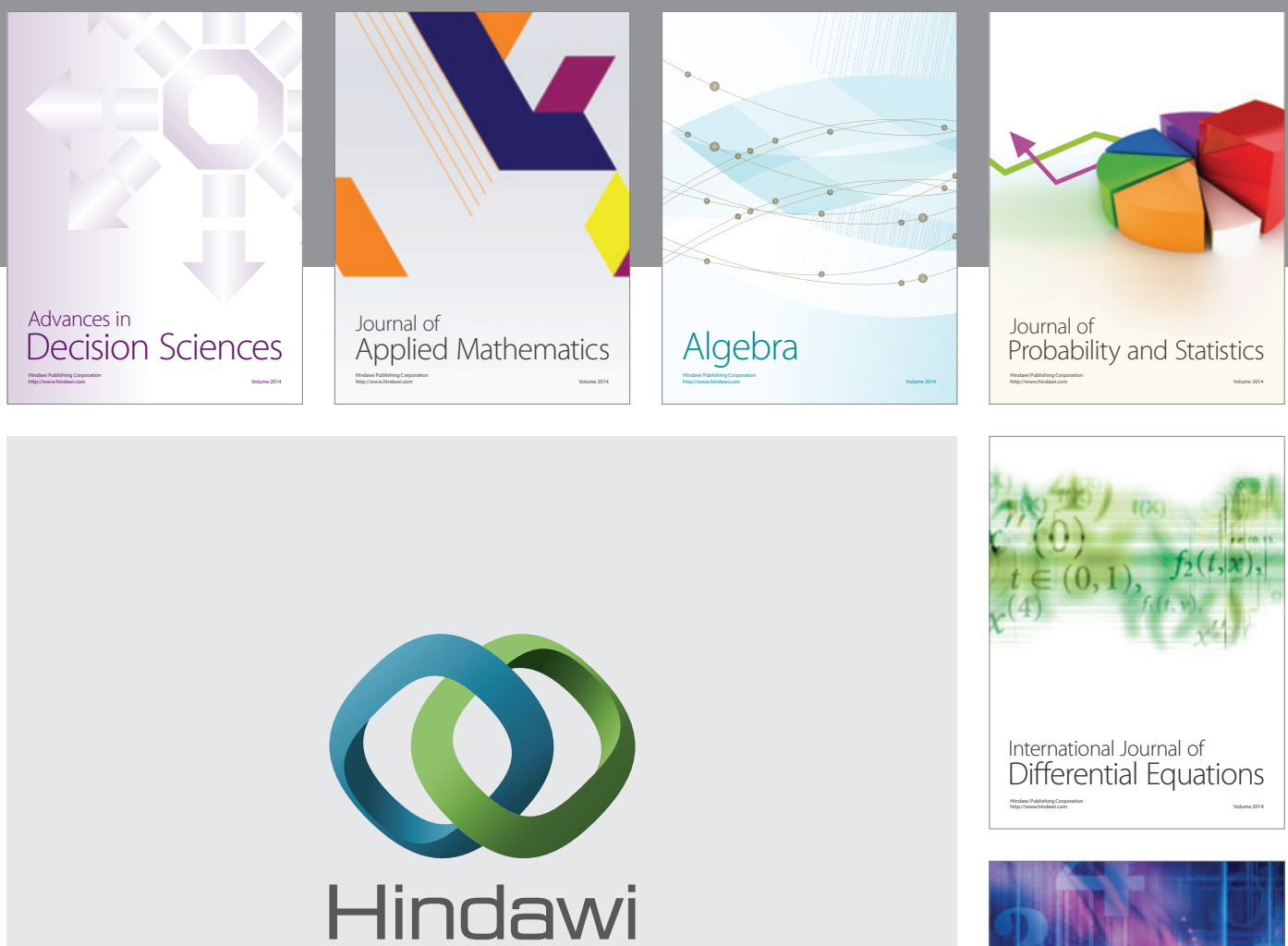

Submit your manuscripts at http://www.hindawi.com
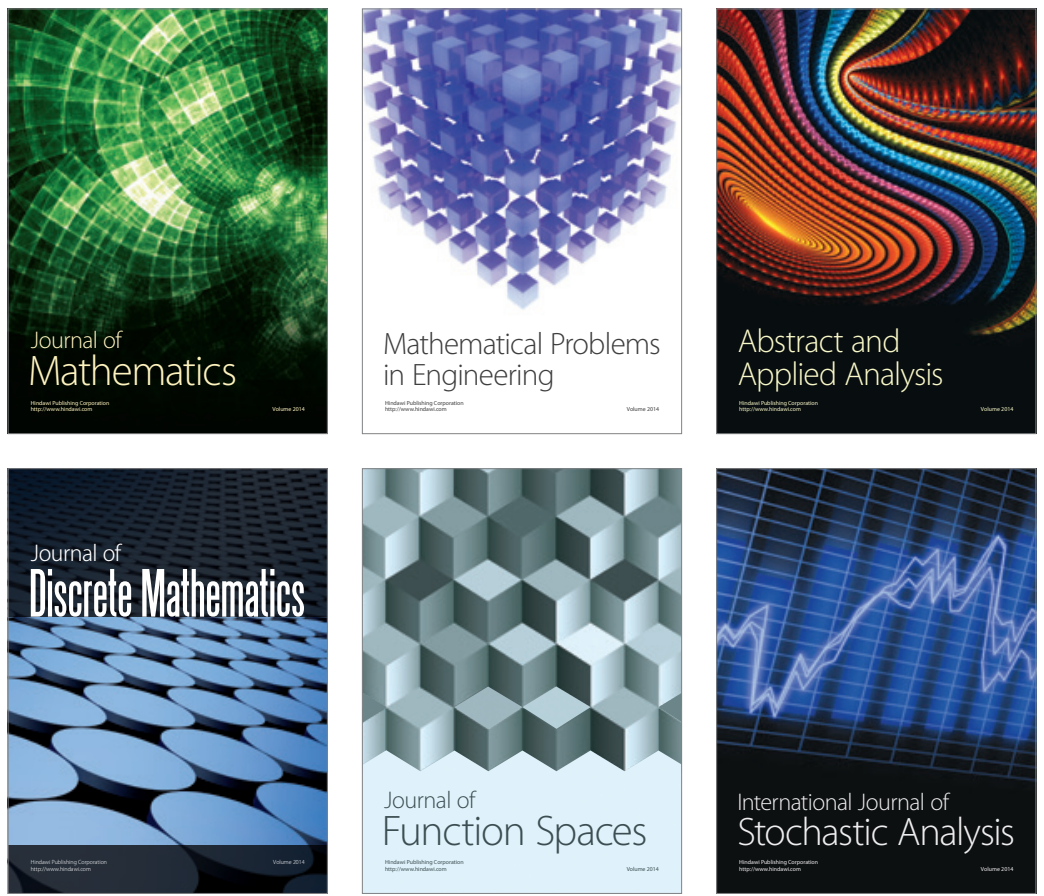

Journal of

Function Spaces

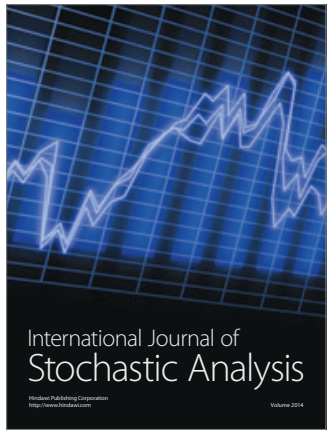

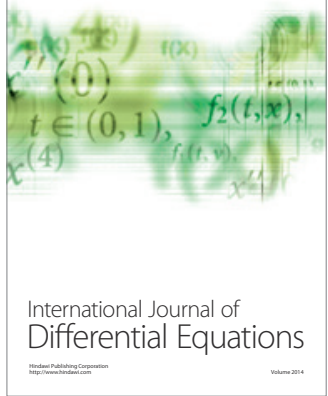
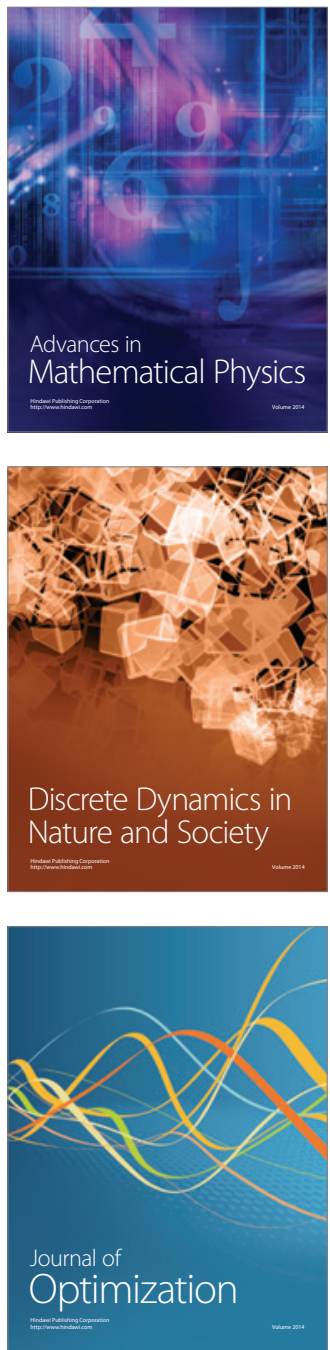